\title{
Foetal development of the human gluteus maximus muscle with special reference to its fascial insertion
}

\author{
Y. Shiraishi ${ }^{1}$, Z.W. Jin' ${ }^{2}$, K. Mitomo ${ }^{1}$, M. Yamamoto ${ }^{1}$, G. Murakami ${ }^{1}$, ${ }^{3}$, H. Abe ${ }^{4}$, J. Wilting ${ }^{5}$, S. Abe ${ }^{1}$ \\ ${ }^{1}$ Department of Anatomy, Tokyo Dental College, Tokyo, Japan \\ 2Department of Anatomy, Wuxi Medical School, Jiangnan University, Wuxi, China \\ ${ }^{3}$ Division of Internal Medicine, Sapporo Asuka Hospital, Sapporo, Japan \\ ${ }^{4}$ Department of Anatomy, Akita University School of Medicine, Akita, Japan \\ ${ }^{5}$ Department of Anatomy, School of Medicine, Georg-August-Universität Götingen, Göttingen, Germany
}

[Received: 6 January 2017; Accepted: 12 June 2017]

The human gluteus maximus muscle (GMX) is characterised by its insertion to the iliotibial tract (a lateral thick fascia of the thigh beneath the fascia lata), which plays a critical role in lateral stabilisation of the hip joint during walking. In contrast, in non-human primates, the GMX and biceps femoris muscle provide a flexor complex. According to our observations of 15 human embryos and 11 foetuses at 7-10 weeks of gestation (21-55 mm), the GMX anlage was divided into 1) a superior part that developed earlier and 2) a small inferior part that developed later. The latter was adjacent to, or even continuous with, the biceps femoris. At 8 weeks, both parts inserted into the femur, possibly the future gluteal tuberosity. However, depending on traction by the developing inferior part as well as pressure from the developing major trochanter of the femur, most of the original femoral insertion of the GMX appeared to be detached from the femur. Therefore, at 9-10 weeks, the GMX had a digastric muscle-like appearance with an intermediate band connecting the major superior part to the small inferior mass. This band, most likely corresponding to the initial iliotibial tract, extended laterally and distally far from the muscle fibres. The fascia lata was still thin and the tensor fasciae latae seemed to develop much later. It seems likely that the evolutionary transition from quadripedality to bipedality and a permanently upright posture would require the development of a new GMX complex with the iliotibial tract that differs from that in non-human primates. (Folia Morphol 2018; 77, 1: 144-150)

Key words: gluteus maximus muscle, iliotibial tract, fascia lata, bipedal gait, human embryo and foetus

\section{INTRODUCTION}

The gluteus maximus muscle (GMX) is the largest skeletal muscle in the human body. This muscle forms a flexor complex with the biceps femoris in non-human primates [9]. In contrast, the human
GMX is characterised by its strong insertion to a thick lateral fibrous band beneath the fascia lata, i.e., the tractus iliotibialis or iliotibial tract [3,5]. In Figure 1, the fascia lata and iliotibial tract are both removed to show the limited insertion of the GMX to the femur.

Address for correspondence: Z.W. Jin, MD, PhD, Department of Anatomy, Wuxi Medical School, Jiangnan University, 1800 Lihu Avenue, Wuxi, Jiangsu, 214122, China, tel: +86 5108519 7079, fax: +86 5108519 3570, e-mail: zwjin@jiangnan.edu.cn 


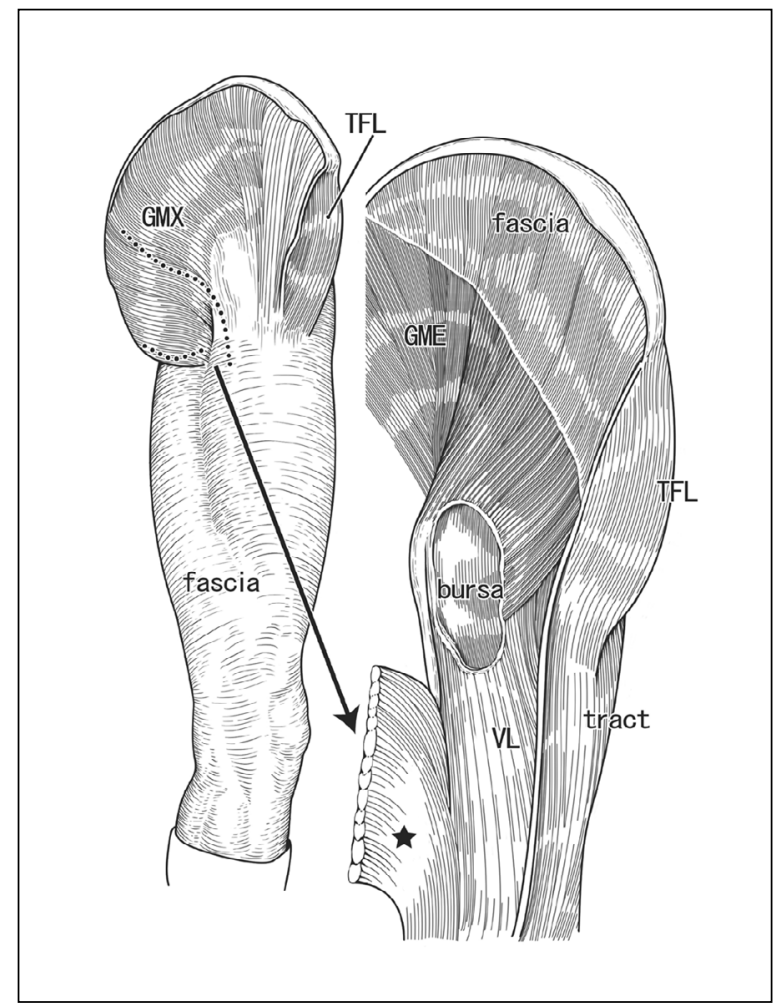

Figure 1. Schematic representation of the human gluteus maximus muscle. The gluteus maximus (GMX) as well as the tensor fasciae latea (TFL) inserts into the iliotibial tract just beneath the fascia lata. In the right-hand part of the figure, the fascia and tract are together removed to show the limited insertion of the gluteus (star) to the gluteal tuberosity of the femur. The inferior and deep one-third of the muscle fibres contribute the femoral insertion. The gluteus medius (GME) inserts to the major trochanter of the femur under the bursa; $\mathrm{VL}$ - vastus lateralis muscle.

With its strong fascial insertion in humans, the GMX plays a major role in maintaining the lateral stability of the pelvis during walking, rather than abduction and/or flexion. In non-human primates, the iliotibial tract may be present but it does not resemble that in humans [4].

According to Bardeen [2], the anlage of the GMX is initially fused with the biceps femoris anlage, and later, the GMX anlage becomes separated into two portions: the distal portion inserting into the femur distal to the great trochanter, and the proximal portion inserting into a fascia over the attachment of the distal portion. His description of two portions similar to the proximal and superficial two-thirds of the adult muscle inserting to the iliotibial tract and the distal and deep one-third inserting to the femur, appears to be imprecise. Bardeen himself described that these two portions are "only rarely" distinct in adults. We have noted that, in the 20-mm crown-rump length (CRL) embryo, a "fascia" receives Bardeen's proximal portion of the GMX. This fascia is unlikely to correspond to the future fascia lata because it is established at 14 weeks, when the specimen is over $115 \mathrm{~mm}$ $C R L$, i.e. much later than the time of thigh muscle development [8]. Another limitation of previous studies might be found in sections to be observed: usual horizontal sections are difficult to show topographical anatomy of the large GMX, but a collection of sagittal sections is few in number in the world.

Consequently, in spite of its critical contribution to the evolutionary transition to bipedality and a permanently upright posture, foetal development of the strong fascial insertion of the GMX does not seem to have been well examined. To provide further clarification of these evolutionary aspects, using sagittal sections, the aim of the present study was to describe the development of the GMX in relation to the iliotibial tract.

\section{MATERIALS AND METHODS}

The study was performed in accordance with the provisions of the Declaration of Helsinki 1995 (as revised in 2013). We used sagittal sections of pelvises and lower extremities obtained from 15 human embryos and 11 foetuses ( 15 males and 11 females) at 7-10 weeks (CRL 21-55 mm). Eight embryos and 8 foetuses were part of a collection kept at the Department of Anatomy, Akita University, Akita, Japan. They had been donated to the Department by the families concerned between 1975 and 1985, and preserved in $10 \% \mathrm{w} / \mathrm{w}$ neutral formalin solution for more than 30 years. The available data were limited to the date of donation and the number of gestational weeks. There was no documentary record of the family name, the name of the obstetrician or hospital, or the reason for abortion. The use of the specimens for research was approved by the University Ethics Committee (No. 1378). The other 7 embryos and 3 foetuses were belonging to Blechschmidt collection in Medical Museum of Georg-August-Universität Göttingen and serial sagittal sections had been prepared. Most sections were stained with haematoxylin and eosin (HE), while a minor part with azan or Masson trichrome staining. The use of these collections did not need a specific approval of the Institute. 
Each of the specimens in Akita, Japan, was cut transversely at a level above the iliac crest. The pelvis was divided into the left and right halves along the midsagittal line. After routine procedures for paraffin-embedded histology, at 20-50 $\mu \mathrm{m}$ intervals depending on the size of the specimens, we prepared semiserial sagittal sections (5-6 $\mu \mathrm{m}$ in thickness) of the pelvis and lower extremity. Most of the sections were stained with $\mathrm{HE}$, while some were used for immunohistochemistry. The primary antibodies used were 1) mouse monoclonal anti-human nestin (1:100; Santa Cruz Biotechnology, Santa Cruz sc23927, CA, USA), and 2) mouse monoclonal anti-human $\$ 100$ protein or S100 (dilution, 1:100; Dako Z0311; Dako, Glostrup, Denmark). Samples were not pretreated by autoclaving because of the loose nature of the foetal tissues. Following incubation with the primary antibody, the sections were incubated with horseradish peroxidase (HRP)-labelled secondary antibody (Histofine Simple Stain Max-PO, Nichirei, Tokyo) for $30 \mathrm{~min}$, followed by incubation with diaminobenzidine (Histofine Simple Stain DAB, Nichirei) for 3-5 min. All samples were counterstained with haematoxylin. We used nestin as a marker of embryonic striated muscle cells $[7,11,12]$. Desmin is well known as a marker of muscle fibres [1], but it is not available for specimens that have been preserved for long periods in fixatives (unpublished data).

\section{RESULTS}

The present observations were based on sagittal sections because our major interest was in the lower or distal extension of the GMX toward the future iliotibial tract. In 7 embryos at 7 weeks (CRL 21-23 mm), the GMX was identified as a single triangular mass of primitive muscle fibres immediately lateral to the anlage of the gluteus medius (Fig. 2). These muscle fibres were positive for nestin (Fig. 2D, F). The medial apex of the triangle was located closely to the ventral aspect of the femur (Fig. 2C, D). The distal or ventral margin of the triangle faced the biceps femoris anlage, but the two were not attached (Fig. 2D). We identified the inferior gluteal nerve supplying the GMX (Fig. 2A). The gluteus medius and iliopsoas were evident, since together they covered and supported the femoral head from the lateral or medial side of the femur, respectively. The short head of the biceps femoris was attached to the peroneal part of the sciatic nerve, while the long head was attached to the tibial part (Fig. 2F). However, these two heads

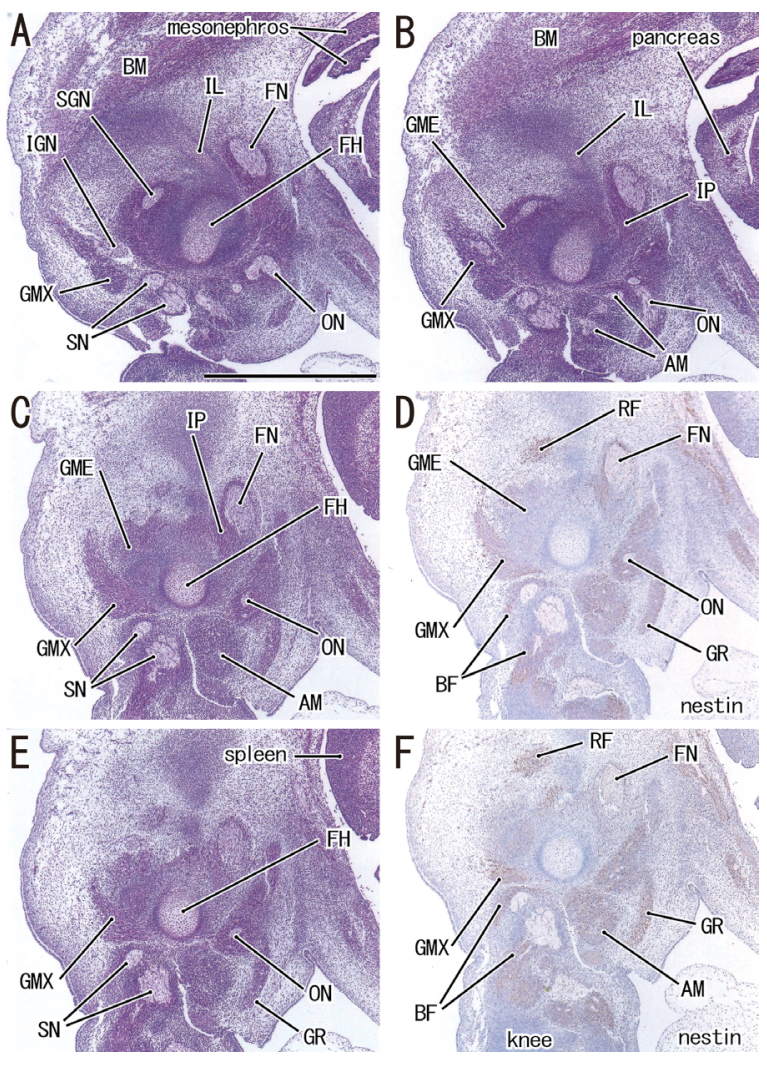

Figure 2. Initial phase of the gluteus maximus as a single triangular mass. Sagittal sections. A specimen at 7 weeks (CRL $21 \mathrm{~mm}$ ). Haematoxylin and eosin (HE) staining (A, C, E) and immunohistochemistry for nestin (D, F). Panel A (or panel F) displays the most lateral (or medial) site in the figure. Intervals between panels are $0.1 \mathrm{~mm}(\mathrm{~A}-\mathrm{B})$ and $0.05 \mathrm{~mm}(\mathrm{~B}-\mathrm{C}, \mathrm{C}-\mathrm{D}, \mathrm{D}-\mathrm{E}, \mathrm{E}-\mathrm{F})$. The gluteus maximus (GMX) can be identified as a single triangular mass, which is located closely to the biceps femoris. The inferior gluteal nerve (IGN) supplies the muscle (A, B). All panels were prepared at the same magnification (scale bar in panel $A, 1 \mathrm{~mm}$ ); $\mathrm{AM}$ adductor magnus muscle; $\mathrm{BF}$ - biceps femoris muscle; $\mathrm{FH}-$ femur head; FN — femoral nerve; GME — gluteus medius muscle; GR — gracilis muscle; IGN — inferior gluteal nerve; IP — iliopsoas muscle; $\mathrm{RF}$ - rectus femoris muscle; $\mathrm{SN}$ - sciatic nerve.

could not be discriminated on the basis of muscle fibre morphology such as a direction of fibres. In contrast to the thick nerves, the ilium, pubis and ischium were still thin.

In 8 embryos at 8 weeks (CRL 24-28 mm), the GMX was clearly separated from the gluteus medius by a loose space (Fig. 3). Being similar to the adult morphology, the GMX provided a thick and bulky plate beneath the gluteal skin (Fig. 3B). The superior extension also appeared to originate from the developing lumbosacral aponeurosis (Fig. 3A). However, in contrast to the adult morphology, the GMX was accompanied by an inferior round mass adjacent to, or 

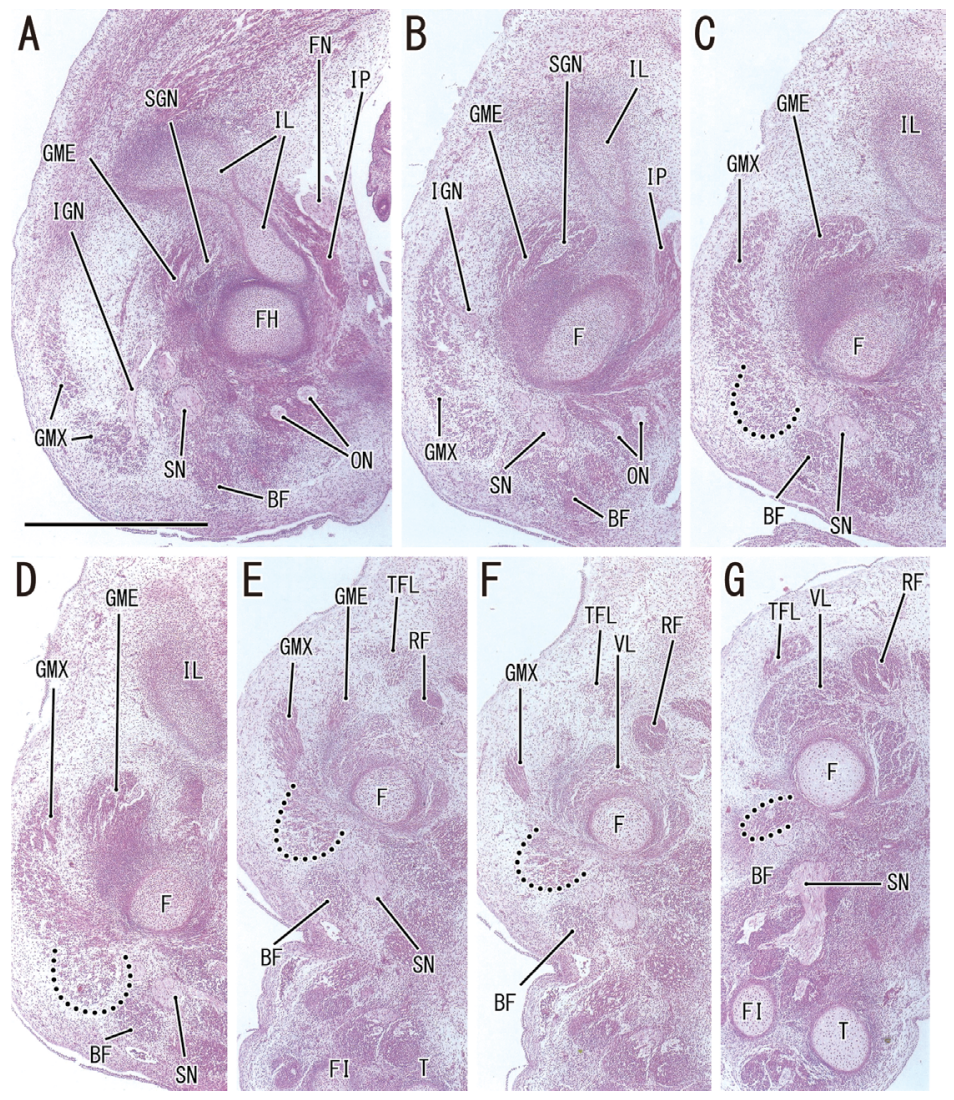

Figure 3. Inferior muscle mass of the gluteus maximus. Sagittal sections. A specimen at 8 weeks (CRL $24 \mathrm{~mm}$ ). Haematoxylin and eosin (HE) staining. Panel A displays the most proximal site in the figure, while panel $\mathbf{G}$ the distal site. Intervals between panels are $0.1 \mathrm{~mm}(\mathrm{~A}-\mathrm{B}, \mathrm{B}-\mathrm{C}), 0.05 \mathrm{~mm}(\mathrm{C}-\mathrm{D}, \mathrm{D}-\mathrm{E}, \mathrm{E}-\mathrm{F})$ and $0.1 \mathrm{~mm}(\mathrm{~F}-\mathrm{G})$. The gluteus maximus can be identified as a major, plate-like mass (GMX) with an additional inferior small mass (partly encircled by the dotted line): these two parts are supplied by two branches of the inferior gluteal nerve (IGN; A, B). Insertions of the iliopsoas (IP) and gluteus medius (GME) together cover the femur (B, C). The tensor fasciae latae (TFL) can be seen (E-G). All panels were prepared at the same magnification (scale bar in panel A, $1 \mathrm{~mm}$ ); BF - biceps femoris muscle; F — femur; FH — femur head; IL — ilium; $\mathrm{ON}$ - obturator nerve; $\mathrm{RF}$ - rectus femoris muscle; $\mathrm{SN}$ - sciatic nerve; VL — vastus lateralis muscle. even continuous with, the biceps femoris (Fig. 3D, E). This inferior muscle mass was independent of, and separated from, the major part of the GMX in medial planes including the rectus femoris (Fig. 3F, G). At 8 weeks, both the major part and the inferior mass were inserted into the femur, possibly forming the future gluteal tuberosity. The inferior gluteal nerve supplied both the major part and the inferior mass of the GMX (Fig. 3A, B). The gluteus medius and iliopsoas carried thick tendinous insertions covering the head of the femur. The major trochanter became evident during this period (Fig. 3B). Beneath the gluteal skin, the tensor fasciae latae was identified as a small muscle mass adjacent to the medial aspect of the gluteus medius (Fig. 3F), being clearly separated from the GMX. The initial tensor fasciae latae appeared to be floating in the subcutaneous tissue without insertion. The muscle fibre morphology appeared to be still the same between the short and long heads of the biceps femoris.

In 11 foetuses at 9-10 weeks (CRL 40-55 mm), the gluteus medius tendon as well as the major trochanter was always thick and strong (Figs. 4, 5). In contrast, most of the original femoral insertions of the
GMX (the major superior part and the inferior mass) appeared to be detached from the femur. Thus, the major part of the GMX was connected to the inferior mass by a musculotendinous band in two specimens (Fig. 4D, E). An intermediate tendon-like structure was seen between the major part and the inferior mass of the GMX in 7 of the 11 specimens (Fig. 5B, E). The thickest part of the connecting band was not attached to the major trochanter but located more distally. The inferior mass of the GMX was adjacent to the short head (Figs. 4B, 5B). The biceps short head was composed of muscle fibres directing distally and inserting to a single thin tendon that was similar to a unipennate muscle, while the long head contained multiple intramuscular tendons that joined together to form a single thick tendon directing proximally. The intermediate tendon or connecting band of the GMX was likely to expand laterally and distally in planes without muscle fibres of the GMX (Fig. 5G). It covered the major trochanter to fix the hip joint as seen in adults. The posterior cutaneous nerve of the thigh appeared to run through the border between the GMX and the semitendinosus or between the GMX and the biceps femoris long head. 
A
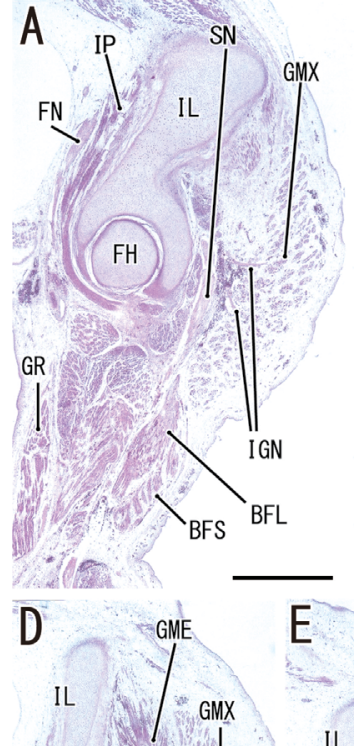

E
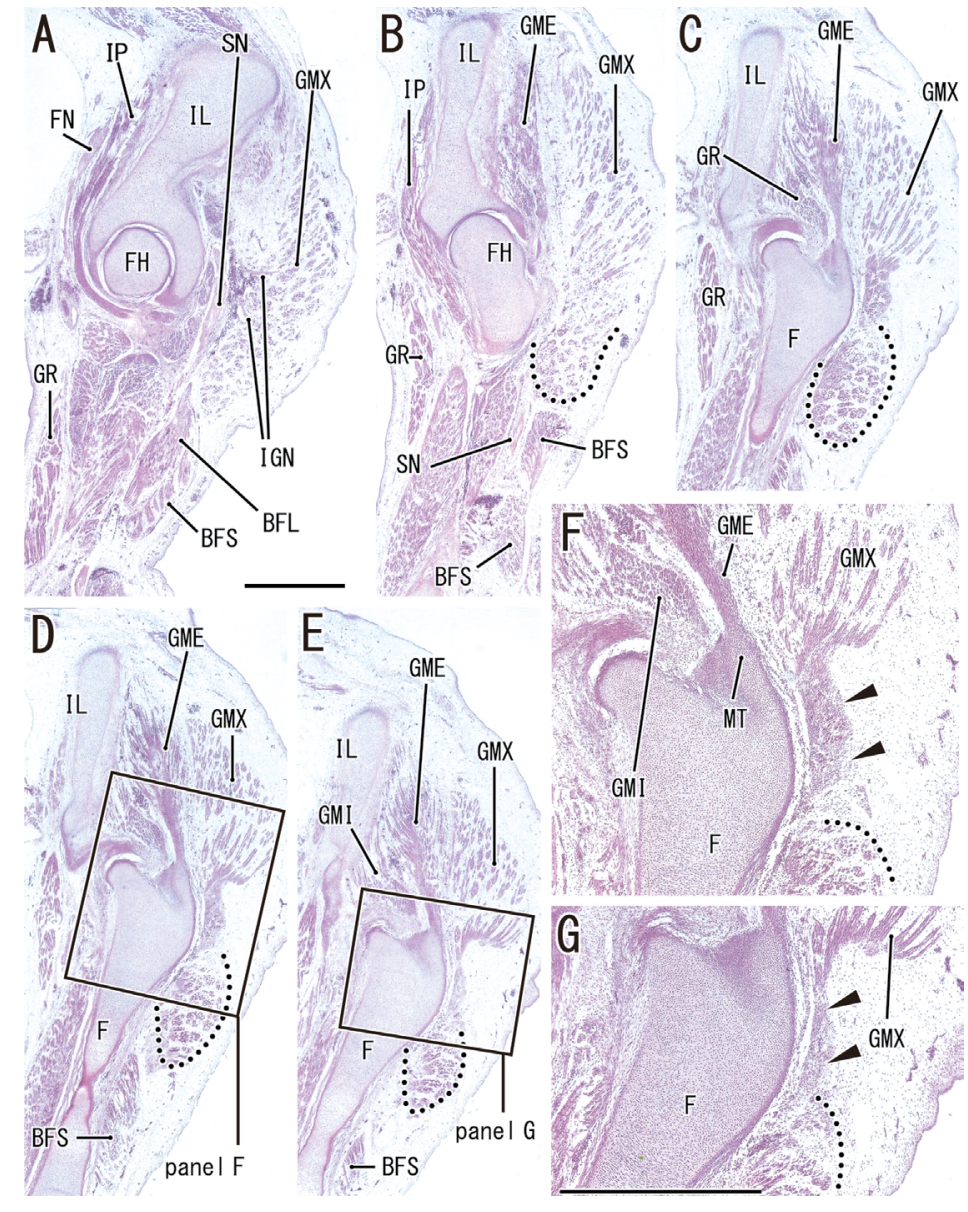

Figure 4. Musculo-tendinous band appears between the major part and the inferior mass of the gluteus maximus. Sagittal sections. A specimen at 9-10 weeks (CRL $42 \mathrm{~mm}$ ). Haematoxylin and eosin (HE) staining. Panel A displays the most medial site in the figure, while panel $\mathbf{E}$ the lateral site. Panels $\mathbf{F}$ and $\mathbf{G}$ are higher-magnification views of the squares in panels $\mathbf{D}$ and $\mathbf{E}$, respectively. Intervals between panels are $0.5 \mathrm{~mm}(A-B), 0.2 \mathrm{~mm}(B-C)$ and $0.1 \mathrm{~mm}$ $(C-D, D-E)$. The major and superior part of the gluteus maximus (GMX) is adjacent to the inferior small mass (partly encircled by the dotted line) in panel $\mathbf{B}$, but these two parts are connected by a musculo-tendinous band in panels $\mathbf{D}$ and $\mathbf{E}$ : the connecting band is indicated by arrowheads in panels $\mathbf{F}$ and $\mathbf{G}$. The inferior mass is adjacent to the biceps long head in panel $\mathbf{B}$ and the short head in panel $\mathbf{B}$. The insertion of the iliopsoas $(\mathrm{IP})$ is shown in panel $\mathbf{A}$, and that of the gluteus medius (GME) in panels C-E. Panels A-E (or panels F and G) were prepared at the same magnification (scale bars in panels $A$ and $G, 1 \mathrm{~mm}$ ); $\mathrm{BFL}$ - biceps femoris long head; BFS — biceps femoris short head; $\mathrm{F}$ - femur; $\mathrm{FH}$ - femur head; FN — femoral nerve; GMI — gluteus minimus muscle; GMX — gluteus maximus muscle; $\mathrm{GR}$ - gracilis muscle; IGN — inferior gluteal nerve; IL — ilium; MT — major trochanter; SN — sciatic nerve.
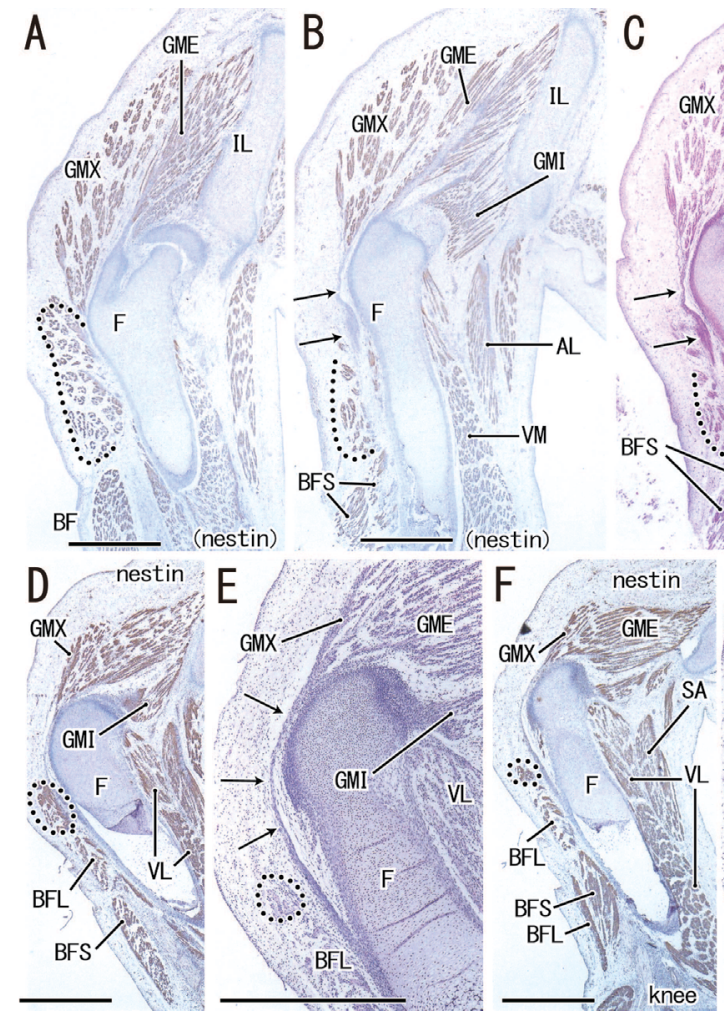
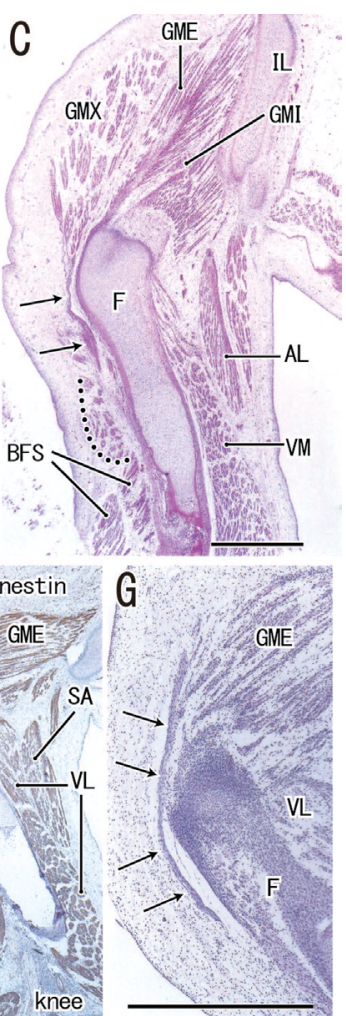

Figure 5. The tendinous band connects the major part and the inferior mass of the gluteus maximus. Sagittal sections. Panels A-C display a specimen at 9-10 weeks (CRL $50 \mathrm{~mm}$ ), while panels D-G exhibit another specimen at 9-10 weeks (CRL $52 \mathrm{~mm}$ ). Haematoxylin and eosin (HE) staining (C, F, H), immunohistochemistry for nestin (A, B, D, F). Panel A or D displays the most medial site in the specimen, and panel $\mathbf{C}$ or $\mathbf{H}$ the lateral site. Intervals between panels are $0.2 \mathrm{~mm}$ (A-B), $0.05 \mathrm{~mm}(B-C), 0.1 \mathrm{~mm}$ (D-E, E-F) and $0.2 \mathrm{~mm}$ $(\mathrm{F}-\mathrm{G})$. The major and superior part of the gluteus maximus (GMX) is adjacent to the inferior small mass (partly encircled by the dotted line) in panel $\mathbf{A}$, but these two parts are connected by a tendinous band (arrows) in panels $\mathbf{B}$ and $\mathbf{C}$. The thickest part of the band is not attached to the major trochanter of femur. In another specimen (D-G), the adjacent two parts of GMX is outside of the figure and we emphasize the connecting band (arrows) extending laterally beyond the GMX as well as the biceps femoris $(\mathbf{H})$. In both specimens, the inferior mass of GMX is adjacent to the biceps short head. All scale bars are $1 \mathrm{~mm}$; $\mathrm{AL}$ - adductor longus muscle; $\mathrm{BF}$ - biceps femoris muscle; $\mathrm{BFL}$ - biceps femoris long head; BFS biceps femoris short head; $\mathrm{F}$ - femur; $\mathrm{GMI}$ - gluteus minimus muscle; IL — ilium; SA - sartorius muscle; $\mathrm{VL}$ - vastus lateralis muscle; $\mathrm{VM}$ — vastus medialis muscle. 

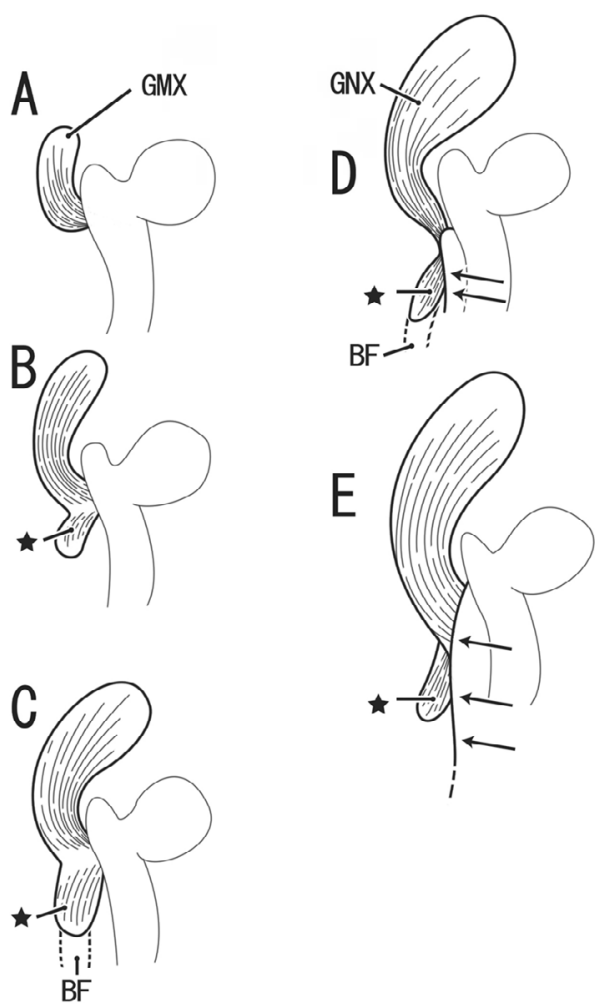

Figure 6. Diagram of foetal development of the gluteus maximus. The gluteus maximus (GMX) is identified as a single anlage until 7 weeks (A). The inferior muscle mass (star) appears at 8 weeks (B), apparently adjacent to or continuous with the biceps femoris (C). A connecting band or intermediate tendon-like structure (arrows) appears between the major superior part and the inferior mass of the GMX (D) and extends laterally far from the muscle fibres (E). We considered this connecting band to represent the initial morphology of the iliotibial band; $\mathrm{BF}$ - biceps femoris muscle.

Figure 6 summarises the present observations. The GMX was first identified as a single anlage by 7 weeks. An additional, inferior muscle mass appeared at 8 weeks, and was adjacent to or continuous with the biceps femoris. A connecting band or intermediate tendon-like structure developed between the major superior part and the inferior mass of the GMX, and expanded laterally far from the muscle fibres. This connecting band appeared to be a candidate representing the initial morphology of the iliotibial band.

\section{DISCUSSION}

In the present study using sagittal sections, we found that the foetal GMX was divided into a major proximal part and a small inferior or distal mass. Since both parts carried bony insertions to the femur at 8 weeks in the reference, our observations might not correspond to the two portions described by Bardeen [2]: a proximal portion with a fascial insertion and a distal portion inserting to the femur (see 'Introduction'). He considered the distal portion or femoral insertion to be the femorococcygeus muscle, which is very common in lower mammals. In the present study, the small distal part seemed to develop later from the major part (Fig. 6B). We found no evidence that the distal mass extended toward the coccyx. Likewise, the sacrotuberous ligament was not developed in the present specimens.

An intermediate tendon-like structure developed between the major superior part and the inferior mass of the GMX (Fig. 6D). This connecting band seemed to differ in terms of development from the well-known intermediate tendons of the digastricus and omohyoideus muscles: the former is provided by a random meeting between two muscles of quite different origin, while the latter results from a change from a single band-like muscle [10]. The laterally-developing major trochanter of the femur was likely to push and extend the intermediate tendon or connecting band in the GMX. However, the thickest part of the connecting band did not attach to the major trochanter, and was located more distally. The inferior mass might be pulled down by the biceps femoris. Likewise, a growth in length of the femur might also contribute to the distal extension of the connecting band.

Notably, the connecting band in the GMX became longer and wider, to extend laterally far from the muscle fibres (Fig. 6E). We considered the connecting band to represent the initial morphology of the iliotibial band. In fact, the connecting band covered the major trochanter to fix the foetal hip joint. Although not shown well in Figure 6, the later-developing inferior mass maintained part of the femoral attachment. To some degree, the inferior mass might produce the insertion to the gluteal tuberosity in adults. We failed to clarify details of further development because of the limited number of sagittally-sectioned specimens. Development of the tensor fasciae latae seemed to occur later than formation of the iliotibial tract from the GMX. In fact, the initial tensor fasciae latae appeared to be floating in the subcutaneous tissue without any insertion. Development of the fascia lata seemed to require an increase in the volume of the thigh muscles, which occurs later than 12 weeks [8]. The 
iliotibial tract and fascia lata overlap in adults, but their developmental mechanisms seemed to differ.

The later-developing, inferior part of the GMX was likely to contribute considerably to initial development of the iliotibial tract, while overgrowth of the gluteus medius seemed to provide the tensor fasciae latae, the latter developing a new insertion to the iliotibial tract. Likewise, the highly developed gluteus medius in humans seemed to provide the large major trochanter, the latter likely contributing to the expanding iliotibial tract. The evolutionary transition from quadripedality to bipedality and a permanently upright posture requires not only the iliotibial tract but also its upward strong traction by the GMX. Conversely, the GMX loses its complex with the biceps femoris and/or muscles around the coccyx. The highly developed GMX appears to require a new strong origin from the sacrotuberous ligament. In fact, the ligament develops in the late foetal stage, in contrast to the sacrospinous ligament [6]. An increase in the volume of the foetal gluteal muscles might be the most essential feature of this evolutionary transition.

\section{CONCLUSIONS}

The foetal GMX had a digastric muscle-like appearance and, later, the intermediate band extended distally: the was be an initial morphology of the iliotibial tract.

\section{Acknowledgements}

We are grateful to Mr. H.G. Sydow for his assistance with the Blechschmidt collection, Goettingen.

\section{REFERENCES}

1. Abe S, Rhee Sk, Osonoi M, et al. Expression of intermediate filaments at muscle insertions in human fetuses. J Anat. 2010; 217(2): 167-173, doi: 10.1111/j.14697580.2010.01246.x, indexed in Pubmed: 20500537.

2. Bardeen RC. Development and variation of the musculature of the inferior extremity and of the neighboring regions of the trunk in man. Am J Anat. 1907; 6: 332-336.
3. Eng CM, Arnold AS, Lieberman DE, et al. The capacity of the human iliotibial band to store elastic energy during running. J Biomech. 2015; 48(12): 3341-3348, doi: 10.1016/j.jbiomech.2015.06.017, indexed in Pubmed: 26162548.

4. Eng CM, Arnold AS, Biewener AA, et al. The human iliotibial band is specialized for elastic energy storage compared with the chimp fascia lata. J Exp Biol. 2015; 218(Pt 15): 2382-2393, doi: 10.1242/jeb.117952, indexed in Pubmed: 26026035.

5. Gerlach UJ, Lierse W. Functional construction of the superficial and deep fascia system of the lower limb in man. Acta Anat (Basel). 1990; 139(1): 11-25, indexed in Pubmed: 2288185.

6. Hayashi S, Kim JiH, Rodriguez-Vazquez JF, et al. Influence of developing ligaments on the muscles in contact with them: a study of the annular ligament of the radius and the sacrospinous ligament in mid-term human fetuses. Anat Cell Biol. 2013; 46(2): 149-156, doi: 10.5115/ acb.2013.46.2.149, indexed in Pubmed: 23869262.

7. Jalouli $M$, Lapierre LR, Guérette $D$, et al. Transitin is required for the differentiation of avian QM7 myoblasts into myotubes. Dev Dyn. 2010; 239(11): 3038-3047, doi: 10.1002/dvdy.22448, indexed in Pubmed: 20931647.

8. Jin $Z W$, Abe $H$, Jin $Y$, et al. Positional changes in tendon insertions from bone to fascia: development of the pes anserinus and semimembranosus muscle insertion in human foetuses. Folia Morphol. 2016; 75(4): 503-511, doi: 10.5603/FM.a2016.0020, indexed in Pubmed: 27830876.

9. Kaseda M, Nakamura M, Ichihara N, et al. A macroscopic examination of $\mathrm{M}$. biceps femoris and $\mathrm{M}$. gluteus maximus in the orangutan. J Vet Med Sci. 2008; 70(3): 217-222, indexed in Pubmed: 18388419.

10. Katori Y, Hyun Kim Ji, Rodríguez-Vázquez JF, et al. Early fetal development of the intermediate tendon of the human digastricus and omohyoideus muscles: a critical difference in histogenesis. Clin Anat. 2011; 24(7): 843-852, doi: 10.1002/ca.21182, indexed in Pubmed: 21538565.

11. Mokrý J, Nĕmecek S. Immunohistochemical detection of intermediate filament nestin. Acta Medica. 1998; 41(2): 73-80, indexed in Pubmed: 9729640.

12. Sjöberg G, Jiang WQ, Ringertz NR, et al. Colocalization of nestin and vimentin/desmin in skeletal muscle cells demonstrated by three-dimensional fluorescence digital imaging microscopy. Exp Cell Res. 1994; 214(2): 447-458, doi: 10.1006/excr.1994.1281, indexed in Pubmed: 7925640. 